\title{
DESARROLLO DEL CONOCIMIENTO SOCIOPERSONAL E INTERVENCION EDUCATIVA
}

\author{
Alfredo Goñi Grandmontagne \\ Universidad del País Vasco
}

\begin{abstract}
RESUMEN. La redacción de este trabajo nos brinda la oportunidad de presentar conjuntamente los resultados de distintos trabajos de investigación, alguno de ellos inédito por el momento, sobre el desarrollo del conocimiento sociopersonal, que hemos venido realizando desde hace varios años. Las premisas de partida de esta línea de investigación derivan del enfoque cognitivo-evolutivo del conocimiento social y, más en particular, de la teoría de los dominios del conocimiento social iniciada por Turiel (1983). El interés de esta teoría no se limita, a nuestro juicio, al importante conjunto de trabajos que ha impulsado; lo más sugerente y prometedor es relacionarla con determinadas preocupaciones básicas que de forma permanente han inquietado no sólo a la psicología sino a todo el pensamiento occidental (Goñi, 2000a). Es entonces cuando se vislumbran los rasgos de un modelo del desarrollo del individuo en la sociedad, al que denominamos personalismo sociomoral, que permite, a la vez que ir integrando los resultados de la investigación, establecer nuevas hipótesis de búsqueda y proponer criterios de intervención educativa.
\end{abstract}

Palabras clave: conocimiento social; desarrollo cognitivo; educación sociopersonal; convenciones sociales; privacidad.

ABSTRACT. This paper offers us the opportunity of showing together the findings of different investigations, some of them unpublished, about the development of sociopersonal knowledge made during the last years. The premises of these investigations come from the developmental-cognitive approach of social knowledge, and specially from Turiel's theory the social knowledge domains.

The richest idea of this working line is to connect it with some concerns that have disturbed not only to Psychology but to occidental thinking either. This is the way as we can glimpse a developmental model of a person in the society which we name sociomoral personalism. It allows us to make up the findings of the investigation, to set up new hypothesis to propound educational contributions.

El conocimiento sociopersonal se refiere a los modos de entender la vida, a las teorías que las personas vamos construyendo, a partir de las numerosas y variadas interacciones con los demás, sobre las relaciones individuo/sociedad, sobre el orden moral, sobre las normas sociales de convivencia y sobre el ámbito privado de decisión individual (Goñi, 1996a). Dicho conocimiento se sustenta, en primer lugar, en una adecuada intelección de las demandas de distinta índole que interpelan a la persona desde su derecho a un proyecto individual y privado de felicidad, desde la necesidad de convivencia civilizada y desde un compromiso ético con la sociedad de la que forma parte. Plantea, en segundo lugar, el problema de cómo articular no sólo el derecho a la privacidad 
con el compromiso ético sino ambas exigencias, a su vez, con una adecuada percepción de las pautas de sociabilidad dentro de una convivencia civilizada. Y, por último, se enfrenta a la cuestión de cómo delimitar las fronteras entre los ámbitos de privacidad, convivencia y moralidad (Goñi, 1996b).

No es preciso subscribir la tesis de Turiel (1983) de que el conocimiento moral, el convencional y el personal constituyen tres dominios distintos de conocimiento social con trayectorias evolutivas independientes y asincrónicas (primera formulación de la teoría de los dominios de conocimiento social, posteriormente matizada por el propio Turiel). Mantenemos, en cambio, que se trata de tres grandes categorías de pensamiento, de tres nociones básicas, de cuyo desarrollo y coordinación derivan modos peculiares y diferenciados de entender la vida (Goñi, 1999).

Lo ideal es que las personas desarrollemos un profundo sentido de justicia, una clarividente percepción del papel de las reglas de convivencia social y un hondo aprecio por el ámbito privado de vida. De la coordinación de estas tres necesidades, para la que no cabe propugnar una fórmula única universal, depende un modelo del desarrollo humano, al que podría denominarse personalismo sociomoral, que va a permitir no caer en las trampas del individualismo autista y asocial (Goñi, 2000a).

No es éste el momento más adecuado para abordar la temática del desarrollo moral (el sentido de justicia); recientes revisiones (cf. p.e. Pérez Delgado y Mestre, 1999) sobre el actual estado de la cuestión nos eximen de ello máxime teniendo en cuenta que no ha sido ésta un asunto al que directamente hayamos dedicado atención expresa en nuestro trabajo de investigación.

Lo que corresponde ahora presentar es la investigación sobre otras tres cuestiones complementarias: la comprensión de la vida privada (conocimiento personal), la comprensión del orden social (conocimiento socio-organizativo), y los problemas que plantea la coordinación de las tres grandes categorías del conocimiento sociopersonal (moral, societal y personal).

\section{El dominio de conocimiento personal}

Uno de los tres dominios de conocimiento social es el del conocimiento personal (Smetana, 1982; Turiel, 1983; Killen, 1985), es decir, aquél que versa sobre las conductas sobre las que cada cual puede decidir en función de apetencias o preferencias individuales, incluso aun cuando pueda derivarse algún perjuicio para uno mismo (lo que configura el espacio de comportamiento prudencial dentro del ámbito personal).

Apenas se ha investigado sobre cómo se construye esta noción de privacidad personal, lo cual no deja de sorprender ya que, a nuestro juicio (Goñi, 1999), se trata de una noción central en el pensamiento moderno. Una parte básica de lo privado, como ha quedado dicho, la configuran un conjunto de conductas que tienen que ver con el comportamiento prudente o imprudente. Y a explorar qué posibles pautas evolutivas experimenta el juicio prudencial dedicamos, hace algunos años, nuestros esfuerzos

Veamos de ofrecer de forma resumida los resultados de nuestro intento de identificar posibles cambios asociados con la edad, entre los 7 y los 16 años, en el razonamiento acerca de las conductas de las que derivan consecuencias nocivas/beneficiosas para quien las realiza. La información obtenida permite formular algunos criterios a tener en cuenta en la educación sociopersonal; los indicaremos aun cuando lo más interesante es integrarlos dentro de una perspectiva más amplia sobre cómo engarzar el proyecto individual de felicidad que cada persona tiene derecho a construir a su 
medida con los compromisos éticos y de convivencia social. Coincidimos en esto con quienes entienden que la ética como amor propio forma una parte tan sustantiva del hecho moral como la ética del deber (Goñi, 1996b y 2000a).

\subsection{El desarrollo de las ideas sobre el perjuicio/beneficio propio}

La temática del razonamiento prudencial apenas cuenta con investigación previa, salvo dos trabajos pioneros de Nucci (198l) y de Tisak y Turiel (1984), y sin embargo es considerable la importancia que los aspectos prudenciales tienen en numerosas situaciones de la vida social y, en concreto, de la vida social escolar (Goñi, 1989b).

Dedicamos un trabajo (Goñi, Bacaicoa y Aguirre, 1995) a estudiar qué características reviste la construcción de la noción de prudencia, como criterio de conducta, utilizando como estímulos que suscitasen la reflexión, comportamientos (acciones y omisiones) imprudentes más bien simples o descontextualizados (fumar, beber, falta de higiene, hacer el vago, no esforzarse... en abstracto) en los que el principal perjudicado es quien los realiza. Un segundo estudio (Goñi, 1993a) planteó situaciones de doble alternativa entre el perjuicio/beneficio propio y otros valores sociales, o entre diversas clases de perjuicio/beneficio personal: ¿cómo articulan sujetos de diferentes edades sus juicios prudenciales con otros juicios sociales?, ¿cuáles son las compensaciones que les inducen a preferir en ocasiones el perjuicio propio o la renuncia a ciertos beneficios?, ¿a cambio de qué no importa perjudicarse a sí mismo? En un tercer informe (Goñi, Aguirre y Bacaicoa, 1997) hemos analizado el razonamiento sobre el comportamiento prudente versus el comportamiento imprudente.

En esas publicaciones pueden consultarse los pormenores técnicos de la investigación. Lo que ahora nos interesa es ofrecer una síntesis de resultados, una visión de conjunto de la información obtenida. Y la resumimos en los siguientes puntos.

1.1.1. Los modos de pensar prudenciales se tornan con la edad más complejos al irse tomando en consideración más variables que sólo el perjuicio propio. La mayoría de los preadolescentes (de 13 y 16 años) ofrecen un análisis del comportamiento imprudente y de sus condicionantes que, en cambio, es infrecuente en los niños de menor edad (7 y 10 años). Hasta los 10 años de edad a muy pocos niños les resulta fácil emplear una argumentación plural mientras que a partir de los 13 años aumenta significativamente el número de quienes apelan a más análisis que el del perjuicio propio (Goñi, Bacaicoa y Aguirre, 1995). Aunque no justifiquen las imprudencias entienden mejor sus causas, tienden a sopesar los pros y contras de la conducta y buscan explicaciones pluricausales del comportamiento. La forma de razonar, a partir de los 13 años, gana además considerablemente en formalización.

1.1.2. Con la edad, y como muestra del desarrollo cognitivo, se produce una progresiva habilidad de coordinar el razonamiento prudencial con otro tipo de juicios sociales al revolver dilemas socioprudenciales. Queda dicho que con la edad se va abandonando la perspectiva puramente prudencial al enjuiciar acciones/omisiones que acarrean perjuicios para uno mismo. Estas pasan a ser percibidas como estímulos complejos que suscitan referencias a la privacidad, a lo psicológico personal y/o a lo moral. Disminuyen las centraciones en aspectos parciales y aumenta la consideración simultánea de varias dimensiones, aun cuando no siempre se encuentre la fórmula para coordinarlas de forma integrada. 
1.1.3. Evolucionan las ideas sobre la normatividad, punitividad y privacidad del comportamiento imprudente. La investigación previa había detectado que se iniciaba con anteriorioridad a los 10 años una tendencia a preferir los argumentos personales a los prudenciales en referencia a las reglas prudenciales. Ahora podemos afirmar (Goñi, Bacaicoa y Aguirre, 1995) que la mayoría de los preadolescentes reclaman el carácter privado del comportamiento imprudente y rechazan que sea punible; frente a los niños menores de 10 años, los de más edad muestran haber evolucionado de entender que se debería prohibir y castigar el comportarse de forma imprudente a considerar que se trata de una conducta no susceptible de regulación normativa y, por supuesto, no punible. Este cambio de idea aparece asociado con la progresiva vinculación de lo prudente con lo privado. Hay que subrayar, en todo caso, que el rechazo a una regulación social del comportamiento imprudente no significa admitir que esté bien comportante de ese modo; ocurre, sin embargo, que se encuentran dificultades para explicar la índole de la exigencia de no perjudicarse a uno mismo.

\subsubsection{Aparecen peculiaridades diferenciales al razonar sobre acciones impru-} dentes versus imprudencias por omisión. La reflexión sobre ambos tipos de imprudencias comparte algunas características comunes: (a) tanto las acciones como las imprevisiones imprudentes son aprobadas por mayor número de personas a medida que aumenta su edad; (b) se incrementa también con la edad el número de quienes entienden que tanto las acciones como las imprevisiones no son punibles y que pertenecen al ámbito de la privacidad. Es decir, que a partir de los 13 años de edad la mayoría de los sujetos entiende, a diferencia de los menores de 10 años, que la imprevisión (el no ganar), al igual que la comisión de imprudencias (el perder), es una conducta privada y no susceptible de castigo.

Pero son también importantes las muchas peculiaridades diferenciales entre el razonamiento que suscitan los perjuicios y el razonamiento sobre la no obtención de beneficios (Goñi, Aguirre y Bacaicoa, 1997). Con independencia de la edad, la imprevisión aparece como una conducta menos reprobable, más compleja, menos exigible, de menor implicación moral y de mayor conexión con el autodesarrollo personal que la imprudencia activa. Luego, por todo ello, decidir no ganar o no beneficiarse pertenece de una forma mucho más nítida aún que la decisión de perder o de resultar perjudicado al ámbito de la privacidad individual.

1.1.5. Con la edad se va enriqueciendo la concepción de lo que beneficia a uno mismo al incluirse dentro del beneficio personal no sólo logros materiales sino también el autodesarrollo o autorrealización personal. A partir de los 10 años aumenta significativamente el número de personas que incluye dentro de la noción de beneficio logros que tienen que ver con el superarse uno mismo, con la consecución de las metas propuestas y con la autoestima. Tal relación se establece más fácilmente (el número total de sujetos que la afirman es mayor y más claro el cambio secuencial asociado con la edad) al reflexionar sobre la pérdida de beneficios que sobre la comisión de acciones perjudiciales (Goñi, Aguirre y Bacaicoa, 1997). Se trata de un referente relevante desde un punto de vista educativo por dos razones básicas: una, porque la importancia de otros beneficios tales como el bienestar físico o el triunfo deportivo tiende a relativizarse con la edad; dos, porque proporciona una pista en clave positiva (logro) para la educación prudencial, siempre preferible a las insistencias en la evitación de perjuicios. 
1.1.6. Varía con la edad la evaluación de unos y otros perjuicios/beneficios de la conducta, lo que condiciona los criterios que se sustentan sobre lo que es prudente y lo que no lo es y, aún más, sobre lo que se debe o no hacer con independencia de que acarrée determinados perjuicios. Estas diferencias interindividuales, asociadas con la edad, aparecen claramente cuando hay que decidir entre elementos alternativos de dilemas socio-prudenciales (Goñi, 1993a). La pertenencia a una cuadrilla aparece, especialmente a partir de los 13 años, como un valor emergente con capacidad de relativizar los perjuicios de ciertas conductas que se realizan cuando se sale con algunas cuadrillas, tales como el rechazo de otros agentes sociales; en cambio se tiene en cuenta el posible rechazo social que pueda causar la suciedad. Pierde atractivo el bienestar físico y el éxito deportivo mientras que se acepta cada vez más, en teoría al menos, el sentido del autodesarrollo.

Pero no sería correcto entender tan sólo que se producen cambios de valores; lo que la mayoría de la gente empieza a entender ya desde los trece años es que el perjuicio/beneficio nunca se da sólo sino que más bien el beneficio implica costos y los perjuicios quizá no sean sino el precio que se paga por una satisfacción inmediata de necesidades. La mayoría de la gente desde los trece años sopesa pros y contras de las conductas con lo que relativiza la importancia de unos perjuicios o beneficios que los niños más pequeños tienden a verlos como absolutos.

1.1.7. Las pautas evolutivas no son la única clave para interpretar la resolución de los dilemas socioprudenciales. La información hasta ahora recogida es consistente con la tesis que explica el desarrollo como una progresiva habilidad de coordinar la argumentación prudencial con otras consideraciones sociopersonales, evolución que guarda relación con un progresivo incremento de la capacidad cognitiva.

Ahora bien, otros datos avalan que en el razonamiento prudencial inciden también otras variables como el contexto socio-cultural, económico, político o ideológico. La definición de lo que perjudica/beneficia tiene que ver, por ejemplo, con el conocimiento experiencial de las situaciones (Goñi, Bacaicoa, Aguirre, 1995) y, en último término, el contenido del comportamiento prudente o imprudente depende del autoconcepto y de la concepción antropológica que se sustente. De ahí que el aprecio por los valores prudenciales experimente cambios en relación con la edad pero probablemente también cambie en función de otras variables como el contexto socio-cultural, económico, político o ideológico; sería impensable buscar para el razonamiento prudencial pautas de evolución de rango universal, al estilo de las propuestas por Kohlberg para el razonamiento moral.

\subsection{Ayudar a construir criterios de prudencia}

Por seguir el estilo telegráfico que exige la limitación de espacio ofrecemos a continuación varias consideraciones de índole psicopedagógica.

1.2.1. Un claro cometido de la educación sociopersonal debería ser el de alentar a construir criterios de prudencia. Hay conductas de marcado carácter prudencial: la higiene, el consumo abusivo de drogas, el mayor o menor esfuerzo en el trabajo, el empleo del tiempo libre... son cuestiones cuya primera referencia no es el perjuicio/beneficio de los demás sino el perjuicio/beneficio de uno mismo. Los programas instruccionales de educación sociopersonal, especialmente en algunas de sus dimen- 
siones (educación para la salud, educación del consumidor, educación ambiental, educación vial...) podrán mejorarse sustancialmente en la medida en que se respeten los patrones evolutivos que experimenta la comprensión del comportamiento prudente e imprudente (Goñi, 1992).

1.2.2. No basta con informar sobre los peligros que acechan a quien se comporta de forma imprudente; lo decisivo es saber cómo procesan los educandos esa información y cómo ayudar a que la sopesen con madurez. Con demasiada frecuencia el esfuerzo de muchos programas de educación para la salud, educación ambiental, educación vial, seguridad e higiene en el trabajo... se agota en una labor informativa. Ahora bien, limitarse a informar sobre riesgos y peligros de la conducta sin atender, ante todo, a los distintos códigos criteriales que las personas activan para procesar dicha información resulta sumamente inadecuado. No basta con advertir de los peligros de determinadas conductas; desde un punto de vista educativo lo decisivo no consiste en advertir de los peligros sino en conocer el auténtico alcance que las personas confieren a un determinado perjuicio y en ayudar a procesar esa información. Y para ello es imprescindible tener en cuenta la forma de pensar de los educandos.

1.2.3. Distinguir, y coordinar, dimensiones de las situaciones socioprudenciales. Los programas de educación socioprudencial deberían prestar particular interés a la discusión de la deontología del comportamiento prudencial: la índole deontológica de estas conductas es distinta de las morales; su obligatoriedad, en caso de existir, se apoya en razones diferentes. Ahora bien, teniendo en cuenta la complejidad conceptual de la mayoría de los problemas socioprudenciales de la vida ordinaria, con frecuencia es preciso además esforzarse por coordinar la reflexión prudencial con otras consideraciones sociopersonales.

1.2.4. Los dilemas socioprudenciales aparen como recurso educativo recomendable para ayudar a construir formas de pensar más elaboradas, dado que resulta más fácil percibir la multidimensionalidad conceptual de las conductas en contexto que en abstracto (Goñi, 1993a).

1.2.5. El periodo de tiempo que va de los 10 a los 13 años de edad es un momento especialmente privilegiado para ayudar a construir modos más elaborados de razonamiento prudencial. Los datos obtenidos mediante entrevistas de inspiración clínica nos permitían sostener que para finales de $2^{\circ}$ de la ESO la mayoría del alumnado ha abandonado su anterior visión infantil acerca del comportamiento prudente e imprudente y que comparte un punto de vista típico de la preadolescencia. Los obtenidos mediante un cuestionario (Goñi, 1994) retrasan algo esta transición y sugieren que las variaciones más significativas tienen lugar entre los 13 y los 14 años. A expensas de poder precisar mejor esta cuestión, lo que queda en claro por el momento es que a lo largo de los años que coinciden con la actual etapa escolar de la Educación Secundaria se va consolidando el perspectivismo prudencial.

De todo lo anterior se infiere que para la mayoría de los niños y niñas los cursos de Educación Secundaria Obligatoria, constituyen un momento privilegiado e insoslayable al cubrir una época de transición entre dos modos distintos de razonamiento 
prudencial. Ese momento resulta especialmente oportuno para poder provocar movilidad cognitiva en el razonamiento prudencial; la intervención educativa debería facilitar el que afloren discrepancias intragrupales a partir de las cuales poder fomentar la discusión y el cambio de perspectivas.

1.2.6. Empezar por trabajar el autoconcepto personal. Contamos con la experiencia de haber sometido a prueba un programa de intervención educativa (Goñi, 1993 b) en orden a fomentar, en alumnado de 1ํ de ESO, criterios de prudencia. Los contenidos y actividades del programa se pusieron al servicio de los siguientes procesos: el análisis de lo que perjudica/beneficia; el fundamento normativo para comportarse de forma prudente; la coordinación con otros juicios sociales. El programa resultó eficaz en cuanto a desarrollo del razonamiento moral pero, sin embargo, no indujo mejoras en el razonamiento prudencial. ¿Por qué? Probablemente porque la reflexión sobre lo que perjudica/beneficia precisa de un previo autoconocimiento que no mostraron los sujetos experimentales.

Todo apunta a que si la argumentación prudencial madura en torno a los 13 o 14 años. Puede que en edades inferiores, incluso en 1ํ de ESO, como fué el caso de nuestra intervención educativa, sea recomendable que la educación sociopersonal se concentre en otras temáticas previas. El conocimiento propio, la autoestima, las habilidades sociales, la clarificación de valores... son otras tantas facetas de la formación personal valiosas en sí mismas y que, de otro lado, resultan imprescindibles para entrar de lleno en la discusión, prudencial, sobre el diseño del proyecto individual de autorrealización humana.

Nos atrevemos, por tanto, a sugerir una propuesta de programación diferenciada para el primero y el segundo ciclo de la ESO. En los dos primeros años merecería la pena insistir en la temática del autoconcepto y de la autoestima dado que sólo a partir de los proyectos individuales de autodesarrollo se puede avanzar en la definición de lo que beneficia y perjudica a cada persona. El segundo ciclo de la ESO se presta mejor al análisis de problemas sociopersonales no sólo desde una perspectiva exclusivamente prudencial sino en combinación con otros criterios (moral, legal, convencional, prosocial...) de conducta.

\subsection{Un campo apenas desbrozado}

Queda aún mucho por explorar sobre los procesos constructivos y las pautas de evolución del conocimiento sociopersonal. Sabemos, eso sí, que las ideas sobre el ámbito privado de libre decisión personal adquieren gran relevancia a partir de la adolescencia; y que difieren en su elaboración, como luego se verá, de unas personas a otras y también de unos contextos culturales a otros. ¿Por dónde proseguir en la indagación? Dos vías presentan, a nuestro juicio, particular relevancia.

Cabe pensar, de un lado, que lo que se manifiesta con fuerza en la adolescencia ha debido irse fraguando con anterioridad y que incluso debe empezar a construirse en épocas tempranas. A este respecto, en un estudio piloto encontramos algunas diferencias interesantes de unos a otros niños y niñas en edad preescolar al enjuiciar si determinadas conductas (vestido, peinado, comer golosinas...) pertenecen o no a su ámbito de libre decisión personal. Y en segundo lugar, son varias las temáticas (sexualidad, elección profesional, proyecto personal de vida...) cuya comprensión guarda relación con las ideas sobre el perjuicio/bene- 
ficio propio y sobre la legitimidad de detraerlas de regulaciones sociomorales. También a este respecto contamos con algunos esbozos sobre cómo convertir estas temáticas en objeto de investigación.

\section{Conocimiento social}

La convivencia social es imposible al margen de códigos que la regulen. Todo grupo social establece sus propios códigos de civilidad, los cuales normativizan las interacciones entre sus miembros. Es sumamente importante entender el sentido de los códigos de interactividad social y, en particular, en qué medida comprometen los Iladamos compromisos sociales; la convivencia civilizada demanda una inteleccción ajustada del peculiar compromiso que merecen los códigos, revisables y alternables, de la organización social.

Una amplia documentación empírica (prácticamente todos los trabajos publicados por Elliot Turiel) ha puesto de relieve que la mayoría de niños y de adultos de numerosos países entienden que asuntos tales como las formas de trato, los modales en la mesa, las expectativas de género y las regulaciones escolares son convenciones sociales. Conceptualizar una práctica social como una convención significa admitir ciertas limitaciones a la libertad de comportamiento propio, pero la obligatoriedad convencional deriva del consenso y es contingente a ese contexto social; en otros contextos pudiera no haber consenso ni obligatoriedad.

En un estudio pionero sobre la comprensión de las convenciones, Turiel (1983) sostiene que las ideas de las personas experimentan sucesivas reorganizaciones evolutivas, en fases de rechazo y aceptación, que culminan en la comprensión de que las convenciones sociales facilitan las interacciones en la vida social. Los datos obtenidos por nosotros mismos (Goñi, 1998), en cambio, inducen a pensar que la comprensión de las convenciones sociales sigue las mismas pautas evolutivas que la comprensión más general del orden social.

\subsection{El papel de los códigos de civilidad}

Nuestro estudio se realizó con cinco grupos (de 32 sujetos cada uno de ellos) de personas de 10, 15, 20, 40 y 65 años de edad. Las diversas hipótesis específicas que formulamos respondían a cuatro expectativas genéricas que se derivan del modelo teórico: 1. Que en la comprensión de los códigos de civilidad se ponen de manifiesto ciertas diferencias asociadas con la edad, y presumiblemente con el desarrollo cognitivo, consistentes en modos más o menos elaborados de comprender la función de los códigos de civilidad en los sistemas sociales; 2. Que dichas diferencias guardan también relación con la formación académica de las personas; 3. Que el desarrollo del conocimiento de los códigos se orienta hacia una mejor coordinación entre la privacidad individual y el carácter convencional de los mismos y hacia una comprensión más rica del papel que ejercen dentro del sistema social; 4. Que las personas no tienen una concepción unitaria de las convenciones sino que discriminan la contribución característica de unos y otros códigos (importancia, raigambre, implicación moral...) al funcionamiento social.

Los resultados permitieron establecer que existen diferencias, asociadas con la edad y con la formación académica, en dos grandes aspectos: tanto en la conceptualización (contenido) como en la argumentación (modos de organizar la informa- 
ción) de los códigos de civilidad. Mientras que unos sujetos los entienden como convenciones sociales justificadas, otros rechazan la necesidad y sentido de que la sociedad convenga en tales prácticas y otros, en fín, distinguen situaciones y coordinan, según supuestos, la privacidad y la convencionalidad. Los modos de razonar, de otro lado, muestran distinta complejidad y elaboración: unos sujetos tratan los códigos como algo socialmente dado, otros entienden mejor cómo funcionan dentro de los sistemas sociales, mientras que un tercer grupo contempla, enjuicia y valora los códigos en referencia al tipo de objetivo social que apoyan. Los resultados dejan también claro que la naturaleza de unos u otros códigos incide en su comprensión facilitando o no su mayor o menor inclusión en lo privado o en lo convencionalmente justificado, facilitando o no una intelección mayor o menor del papel que juegan en la organización social.

¿Qué relación existe entre estos distintos modos de clasificar y de argumentar sobre los códigos de civilidad? Del conjunto de los resultados, y obviando por el momento mayores precisiones y matices, se deduce que:

1. Quienes ofrecen una comprensión más elaborada pertenecen en su mayoría a los grupos de edad de 20 y de 40 años y al grupo de mayor formación académica en comparación con el de formación académica menor: establecen algún tipo de coordinación entre lo privado y lo socialmente convenido; más allá de identificar cómo funcionan los códigos, sopesan y valoran la finalidad social a la que prestan apoyo.

2. El grupo de edad de 15 años muestra un perfil propio y diferente de los demás grupos: parte de este grupo se asemeja al de 10 años, otra parte se parece al de 20/40 años, en tanto que el sector más peculiar ofrece una versión intermedia: tiende a rechazar el sentido convencional de esos códigos, reclama libertad de decisión personal o no coordina ambos aspectos; no cuenta con criterios de valoración muy precisos. Se trata de un grupo con importante variabilidad interna, dato que encierra valor educativo, que luego comentaremos.

3. El grupo de 10 años revela una considerable perplejidad frente a la mayoría de los códigos: identifica algunos (historias del maquillado, modales en la mesa, y desnudo) mientras que puede costarle conocer la existencia de otros. En todo caso, tiende a tratar los códigos como algo dado y en sus aspectos más elementales: lo habitual, lo esperado...

4. El grupo de 65 años resulta peculiar y diferente. Ofrece a veces respuestas que se asemejan al grupo de 10 años: ambos grupos destacan por aceptar las convenciones; pero los argumentos de justificación son distintos: suele apelar a razones que avalan mantener los códigos. De otro lado, es un grupo de notable variabilidad interna.

5. Se constatan importantes variaciones en la interpretación y argumentación que suscitan unos y otros códigos, estableciéndose la línea de demarcación más clara entre los asuntos relativos a modales en la mesa, maquillado y desnudo público de un lado y el tratar de usted y el vestido para ir al teatro de otro lado.

En los dos últimos casos se trata de códigos de civilidad sobre los que no existe, o ha desaparecido, amplio consenso social, por lo que en consecuencia se les otorga escasa relevancia y no se ve que guarden demasiada relación ni con la buena educación ni con posible ofensa ajena; se reclama mayoritariamente que cada cual haga lo que quiera. 
Los tres primeros códigos (y sus contextos), en cambio, se ajustan en buena medida a los rasgos que caracterizan a los códigos socialmente convenidos y aceptados por lo que resultan ser buenos ejemplares de convenciones sociales entendidas como algo establecido (lo normal), algo importante y algo que no se inscribe en el ámbito de los gustos y preferencias de cada cual. En los tres casos se percibe su relación con molestia ajena. Pero es importante destacar que mientras que los modales en la mesa y el desnudo se asocian con la buena educación, en cambio se rechaza la asociación entre mala educación y contravención del modo esperado de apariencia física (maquillado).

Estos resultados admiten una doble discusión: primero en clave de explicación psicológica y luego en la vertiente educativa.

Queda claro que el enfoque evolutivo estructural adoptado ha permitido identificar, siquiera como esbozo, distintos modos de entender las códigos de civilidad; la comprensión de dichos códigos guarda estrecha relación con la comprensión de los sistemas sociales, la cual a su vez mantiene correspondencia con el desarrollo cognitivo, presumiblemente asociado con la edad. Todo esto aboga en favor del constructivismo. Por decirlo de otra forma, los datos obtenidos no encajan en ninguno de los modelos teóricos que desestimen la interpretación que cada sujeto lleva a cabo de la realidad social a partir de las distintas herramientas cognitivas de las que dispone en distintos momentos de su desarrollo evolutivo.

Cuestión diferente es si se necesita postular la existencia de dominios diferentes de conocimiento social. En aras de la parsimonia científica cabe decir que no; lo cual no significa empero que pierda interés la distinción entre moralidad, convencionalidad y privacidad. Queda suficientemente claro que se trata de categorías básicas en torno a las cuales se organiza la comprensión de la vida social y puede postularse, sin necesidad de apelar a la idea de los dominios, su condición de campos de contenido que encierran una peculiar complejidad. Y así como se ha estudiado, y ha resultado revelador descifrar, la dificultad relativa de numerosos contenidos específicos, los datos obtenidos animan a proseguir en el análisis que encierra el aprendizaje de estos conceptos acerca de cuya importancia coinciden las principales ciencias humanas y sociales.

Discrepamos también de Turiel en sus reticencias a aceptar el influjo de las variables socioculturales como mediatizadoras de la construcción del conocimiento social: es evidente el influjo de la formación cultural; y en otros estudios se ha comprobado la incidencia de variables tales como las creencias religiosas y los valores.

Es francamente complicado reclamar un dominio de conocimiento socioconvencional cuando resulta tan complicado encontrar convenciones prototípicas, siendo muy clara la ambigüedad con que el conjunto de la sociedad percibe la naturaleza de tantos códigos.

En un terreno más concreto, el de la comprensión de la vida social, los datos obtenidos avalan afirmaciones que se orientan hacia la idea de que la buena educación es cosa de inteligentes en el sentido de que las personas que muestran una comprensión más desarrollada de los códigos de civilidad se caracterizan por lo siguiente: 1. No rechazan los códigos pero tampoco los aceptan sin más; valoran tanto sus ventajas como sus inconvenientes; 2 . Tratan a los códigos no como fórmulas fijas, no como recetas, sino como recursos técnicos al servicio de una estrategia; se rechaza la idea del manual de recursos y del protocolo rígido, reclamando un cambio una flexibili- 
dad en el uso que conlleva la decisión no sólo de cómo usar la fórmula establecida sino también de cuándo hacerlo.

\subsection{Implicaciones educativas}

Formuladas en plan telegráfico, he aquí algunas de las conclusiones educativas que derivan de lo anterior:

1. No se pueden confundir, ni abordar ni tratar de educar de la misma manera en asuntos que tienen que ver con los derechos de los demás y en asuntos de índole socio-organizativa. Resulta decisivo, por otro lado, respetar el ámbito de privacidad.

2. Los niños de 10 años muestran un importante desconcierto respecto a la naturaleza de los códigos de civilidad (y nos atrevemos a generalizar este estado a todo el conjunto más amplio de códigos de convivencia y organización social). Y sin embargo la sociedad (padres y maestros, en especial) les exige respeto a los mimos.

3. Nos ha sorprendido que ni siquiera los adultos que han mostrado haber captado mejor el sentido de los códigos (aun siendo muchos de ellos universitarios) se hayan visto sobrados de conocimientos históricos y sociológicos al respecto.

4. Nos atrevemos a proponer que, especialmente en la Educación Secundaria, se incorporen unidades didácticas que proporcionen información y fomenten la madurez de pensamiento sobre estas dimensiones del desarrollo sociopersonal.

\subsection{Seguir investigando}

De entre los numerosos aspectos que reclaman una mayor indagación, lo que a nosotros más nos interesa explorar es cómo se entiende el papel de los códigos de civilidad en los sistemas sociales y las relaciones que se establecen entre lo convencionalmente consensuado y la libertad individual de desmarcarse de los códigos con mayor o menor consenso social. ¿Cuáles son los factores que afectan la desigual delimitación entre lo privado y lo convencional? ¿Cómo avanzar en la explicación de por qué unas personas aceptan y otras rechazan el carácter convencional de unos y otros códigos de civilidad?

Arrojaría gran luz sobre la comprensión de las convenciones sociales la realización de estudios transculturales sobre unos mismos códigos de civilidad; en esta línea contamos con una investigación inconclusa que compara los resultados obtenidos con muestras del País Vasco y de México. Pero, de forma paralela, se precisa realizar estudios diferenciados sobre ámbitos convencionales específicos tales como las maneras en la mesa, la apariencia física, los modos de saludo (y de hablar, en general), etc... Otro tema apasionante es el de los códigos particulares de los grupos (por ejemplo, de los juveniles) lo que permitiría precisar si el convencionalismo o el anticonvencionalismo tiende a revestir carácter generalizado o a sustentarse diferencialmente en unos $u$ otros ámbitos de la convivencia social.

Puede centrarse la investigación en identificar dimensiones evolutivas transculturales o generales, pero resulta sugerente preguntarse también qué tiene que ver la aceptación/rechazo de los códigos de civilidad con variables como el dominio de 
habilidades sociales, la necesidad de afiliación o pertenencia grupal, las representaciones sociales, los rasgos de personalidad, o las creencias, valores e ideologías.

\section{Las discrepancias sobre asuntos sociales controvertidos}

No todo el mundo coincide en cuál es el sitio por donde discurren las fronteras entre lo privado, lo convencionalmente regulado y lo intrínsecamente obligatorio. En efecto, a nadie se le escapan las notables discrepancias (interculturales, de una época histórica a otra e incluso interindividuales dentro de una misma cultura) respecto a la naturaleza convencional, moral o privada de determinados sucesos sociales. Encierra gran interés, tanto teórico como social, el explicar por qué resultan tan difíciles, discrepantes y controvertidos los juicios acerca de asuntos socialmente polémicos como pueden ser el aborto, el desnudo público, la eutanasia, la homosexualidad o el consumo abusivo de drogas.

La investigación previa ha probado la fecundidad heurística de tratar de explicar las divergencias sobre asuntos sociales controvertidos como un desigual reconocimiento de la índole moral, convencional o privada de los mismos. Es lo que puso de relieve el estudio pionero de Smetana (1982) sobre las discrepancias al categorizar el aborto como asunto de índole moral o privada y cuyos resultados nosotros mismos (Goñi, 1997b) hemos corroborado. Y es lo que confirman las investigaciones sobre cómo se entienden el consumo de drogas (Nucci y Weber, 1991), el desnudo público (Turiel, 1983), los estereotipos de género (Goñi et al.,1995) o los temas que provocan discusiones en la familia (Smetana, 1998, 1999; Goñi, 1997a y 2000b) para los que los padres reclaman exigencias de funcionamiento sociofamiliar que los hijos adolescentes tienden a rechazar. Cabe igualmente explicar las distintas interpretaciones del divorcio (Goñi, 1997b) como una discrepancia sobre las dimensiones moral, privada y sociorganizativa de los mismos. Y en esta misma línea se inscriben los resultados de Palacios (1999) sobre la comprensión de la desobediencia civil.

En cualquier caso, la mayor o menor delimitación de los ámbitos de privacidad, de sociabilidad y de justicia parece que depende, en definitiva, de la concepción antropológica y sociocultural que se sustente (Salzstein, 1991). Una de las expresiones de dicha concepción se manifiesta en los valores que se sustentan; de ahí, la conexión de la temática del conocimiento sociopersonal con la de los valores (Palacios, 1999).

Nuestros próximos trabajos, actualmente en curso, se centran en indagar, desde esta misma perspectiva, las siguientes temáticas: 1. Las discrepancias entre profesores y alumnos sobre las normas de disciplina escolar; 2. La correspondencia entre la conceptualización (privada o moral) del aborto y determinado perfil de valores (y otras variables sociopersonales) del alumnado universitario; 3. La relación entre concepciones de vida y elección profesional.

\section{El personalismo sociomoral}

Hasta ahora hemos venido indicando diversas temáticas cuyo interés, tanto científico como social, esperamos que haya quedado patente. Ahora bien, el conjunto de todas estas temáticas permite, a nuestro juicio, esbozar los rasgos de un modelo del desarrollo del individuo en la sociedad, al que denominamos personalismo sociomo- 
ral, que se nos brinda como un sugestivo campo de indagación que vamos a tratar de presentar a continuación.

Los modos de entender la vida dependen en buena medida del desarrollo independiente de los tres citados tipos de juicio sociomoral: el personal, el social y el sociomoral. Tal desarrollo permite al individuo discriminar la distinta entidad de unos u otros compromisos sociopersonales. Así, el proyecto individual de felicidad que toda persona tiene derecho a construir a tenor de su idiosincrasia particular va a depender, ante todo, de la madurez con que entienda su derecho a la privacidad y a velar por sus intereses propios. La convivencia civilizada demanda una intelección ajustada del peculiar compromiso que merecen los códigos, revisables y alternables, de la organización social. Y el compromiso social de solidaridad, exigible y generalizable, no puede sustentarse sino en la comprensión profunda de los principios universales de justicia. Pues bien, lo ideal es que las personas desarrollemos un profundo sentido de justicia, una clarividente percepción del papel de las reglas de convivencia social y un hondo aprecio por su ámbito privado de vida.

Pero, llegados a este punto, se plantean una serie de cuestiones de hondo calado: ¿es compatible la creciente demanda individualista de privacidad con el interés debido a la vida social y con el compromiso ético en favor de los demás?, ¿cómo compatilizar entre sí los motivos y valores morales con los intereses y valores personales?

Estamos convencidos de que todo ser humano sostiene, de forma más o menos explícita, fórmulas que le permiten afrontar a diario situaciones de conflicto entre individualismo, sociabilidad y solidaridad. Pero es muy poco lo que sabemos sobre cómo resolvemos las personas la coordinación de semejante tripleta de demandas: privacidad, conveniencia social e imperativo ético.

Hasta ahora, la psicología, influida poderosamente por el enfoque de Kohlberg, ha prestado interés preferencial al proceso mediante el cual las consideraciones morales terminan por prevalecer sobre el pragmatismo y sobre las expectativas grupales. Bueno será que empiece a conceder la debida atención a la cuestión de si cabe entender, en línea con Turiel, el desarrollo del conocimiento sociomoral como la progresiva capacidad de coordinar los juicios coexistentes sobre la justicia, la convivencia y la privacidad; y si, al mismo tiempo, va progresando la capacidad de recurrir a cada uno de estos dominios de juicio, bien por separado o bien combinadamente, según lo demande la naturaleza de cada situación sociopersonal.

Presumimos que el desarrollo sociopersonal se pone de manifiesto a medida que se va pasando de una forma solipsista de entender el beneficio/privacidad a una forma más compatible con el bienestar y derechos de los demás. Como principales hipótesis sostenemos que la madurez del juicio sociopersonal: 1. permite distinguir los compromisos de justicia de los compromisos sociales; 2 . se va expresando a medida que en la definición del bien propio se es capaz de ir incorporando el bienestar de los demás, esto es, a medida que se va pasando de una forma solipsista de entender el beneficio/privacidad a una forma más compatible con el bienestar y derechos de los demás; 3. se refleja en formas más flexibles y ricas de compaginar la privacidad con la sociabilidad.

Aun cuando carecemos aún de corroboración empírica plena, se pueden aportar algunos datos (Goñi, 2000a) que prestan sustento a la idea de que, a lo largo de los años de la adolescencia, el desarrollo normativo viene caracterizado por una progresiva valoración de la privacidad personal, por un creciente sentido crítico de 
los compromisos socioconvencionales y por un juicio moral más autónomo. Al mismo tiempo, irá progresando la capacidad de recurrir a cada uno de estos dominios de juicio, bien por separado o bien combinadamente, según lo demande la naturaleza de cada situación sociopersonal, aunque aún es mucho lo que queda por investigar sobre este proceso.

No tiene justificación alguna, sin embargo, pensar que los juicios sociopersonales se desarrollan necesaria y universalmente sin que importe el tipo de mediación educativa; sucede, más bien, todo lo contrario aun cuando no es este el momento de hablar de ello.

De otro lado, el desarrollo sociopersonal no puede reducirse a la sola construcción mental de la realidad sino que es, al mismo tiempo, una construcción tanto afectiva como relacional. Dicho de otra forma, en el desarrollo sociopersonal inciden un amplio conjunto de procesos tanto cognitivos como afectivos e interrelacionales. Quiere esto decir que nos parece relevante y pertinente el incluir la exploración de más variables que las ideas de los sujetos. En esta dirección se insertan algunos de nuestros trabajos de investigación en curso en los que, por ejemplo, se buscan correlaciones entre los modos de pensar sobre la realidad sociopersonal con características tales como la empatía, la personalidad, la necesidad de afiliación grupal, la prosocialidad, las actitudes hacia el medio ambiente u otras.

\section{Referencias Bibliográficas}

BÉJAR, H. (1988). El ámbito íntimo. Privacidad, individualismo y modernidad. Madrid: Alianza. Camps, V. (1993). Paradojas del individualismo. Barcelona: Crítica.

GABENNESCH, H. (1990). The perception of social conventionality by children and adults. Child Development, 61, 2047-2059.

GEIGER, K., y TURIEL, E. (1983). Disruptive school behavior and concepts of social convention in early adolescence. Journal of Educational Psychology, 75(5), 677-685.

GOÑI, A. (1989a). La comprensión de normas escolares. Investigación en la Escuela, 9, 75-83.

GOÑI, A. (1989b). La conceptualización de la vida social escolar. Infancia y Aprendizaje, 47, 101-116.

GOÑI, A. (1989c). La coordinación de conceptos en el razonamiento sobre situaciones sociales complejas. Boletín del Instituto de Ciencias de la Educación, 14, 5-20.

GOÑI, A. (1989d). Obligatoriedad antropológica de perfección y psicología del desarrollo moral. Revista de Ciencias de la Educación, 139, 331-342.

GOÑI, A. (1992). La educación social. Un reto para la escuela. Barcelona: Graó.

GOÑI, A. (1993a). La resolución de dilemas socioprudenciales. Revista de Ciencias de la Educación, 156, 467-480.

GOÑI, A. (1993b). Un programa de educación sociopersonal atento a la distinción entre prudencia y moralidad. Revista de Psicología de la Educación, 13, 35-50. 
GOÑI, A. (1994). El desarrollo de las ideas sobre lo privado y lo prudente en la preadolescencia. Revista Interuniversitaria de Formación del Profesorado, 21, 221 229.

GOÑI, A. (1996a). La construcción del conocimiento sociomoral. En F. Bacaicoa (Ed.), La construcción de conocimientos (pp. 307-318). Bilbao: UPV/EHU.

GOÑI, A. (1996b). Proyecto individual de felicidad y compromiso social de justicia. Cultura \& Educación, 3, 19-30.

GOÑI, A. (1997a). Adolescencia y vida familiar. El sentido de las discusiones. Bienestar y Protección Infantil, III (1), 121-133.

GOÑI, A. (1997b). Modos de entender el aborto, formas de entender la vida. Revista de Psicología, XIX(2), 29-49.

GOÑI, A. (1997c). Distintas concepciones de la naturaleza sociomoral del divorcio. Revista de Psicología de la Educación, 21, 83-96.

GOÑI, A. (1998). Códigos de civilidad. Un estudio sobre la comprensión de las normas que regulan la convivencia social. (Inédito).

GOÑI, A. (1999). Lo ético en psicología. La cuestión de dominios o ámbitos. En E. Pérez Delgado y V. Mestre (Eds.), Psicología moral y crecimiento personal (pp. 27-42). Barcelona: Ariel.

GOÑI, A. (2000a). Psicologŕa del individualismo. Donostia: Erein.

GOÑI, A. (2000b). Adolescencia y discusiones familiares. Madrid: Eos.

GOÑI, A. (Ed.) (1996). Psicología de la educación sociopersonal. Madrid: Fundamentos.

GOÑI, A.; AGUIRRE, J. J.; y BACAICOA, F. (1997). ¿Da lo mismo perder que no ganar? Revista de Psicología General y Aplicada, 50 (1), 127/136.

GOÑI, A.; ALEJO, E.; y MTZ. DE ICAIA, I. (1997). Privacidad versus moralidad en el juicio sobre el aborto y el divorcio. Revista de Psicodidáctica, 3, 37-56.

GOÑI, A.; BACAICOA, F.; y AGUIRRE, J. J. (1995). La comprensión del comportamiento imprudente. En A. Goñi (Ed.), Psicodidáctica y aprendizajes escolares (pp. 319-338). Bilbao: UPV/EHU.

GOÑI, A.; CELORIO, G.; y MOLERO, B. (1995). Estereotipos de género: convención, normalidad, justicia. Investigación en la Escuela, 26, 83-92.

HELWIG, C.; TISAK, M.; y TURIEL, E. (1990). Children's social reasoning in context: reply to Gabennesch. Child Development, 61(6), 2068-2078.

KILLEN, M. (1985). Children's coordination of moral, social and personal concepts. (Tesis doctoral). Universidad de California: Berkeley.

MILLER, J. C.; y BERSOFF, D. M. (1988). When do american children and adults reason in social conventional terms? Developmental Psychology, 24, 366-375.

NISAN, M. (1988). A story of a pot: or a cross-cultural comparison of basic moral evaluations. A response to the critique of Turiel, Nucci and Smetana (1988). Developmental Psychology, 24(1), 144-146. 
NUCCCI, L.; WEBER, E. K. (1991). The domain approach to values education: from theory to practice. En W. M. Kurtines; y J. L. Gewirtz (Eds.), Handbook of moral behavior and development. Vol.III: Application (pp. 251-266). Hillsdale, New Jersey: Erlbaum.

NUCCl, L. (1981). Conceptions of personal issues: a domain distinct from moral or societal concepts. Child Development, 52, 114-121.

NUCCI, L. P. (1981). The development of personal concepts: a domain distint from moral or societal concepts. Child Development, 52,114-121.

NUCCl, L. P. (1982). Conceptual development in the moral and conventional domains: implications for values education. Review of Educational Research, 52, 93-122.

PALACIOS, S. (1999). La desobediencia civil y la objeción de conciencia. Comprensión sociomoral de sucesos complejos durante la adolescencia y la juventud. Bilbao: UPV/EHU.

SALTZSTEIN, H. D. (1991). Why are nonprototypical events so difficult, and what are the implications for social-developmental psychology? Monografs of the Society for Research in Child Development, 56(2), 104-116.

SHWEDER, R. A.; MAHAPATRA, M.; y MILLER, J. G. (1987). Culture and moral development. En J. Kagan; y S. Lamb (Eds.), The emergence of morality in young children (pp. 1-83). Chicago: University.

SMETANA, J. G. (1982). Concepts of self and morality: women's reasoning about abortion. New-York: Praeger.

SMETANA, J. G. (1983). Social-cognitive development: domain distintions and coordinations. Developmental Review, 3, 131-147.

SMETANA, J. G. (1986). Children's impressions of moral and conventional transgressors. Developmental Psychology, 21(4), 715-724.

SMETANA, J. G. (1988). Adolescents' and parents' conceptions of parental autorithy. Child Development, 59, 321-335.

SMETANA, J. G. (1989). Adolescents' and parents' reasoning about actual family conflict. Child Development, 60, 1052-1067.

SMETANA, J. G. (1991). Adolescents' and mothers' evaluations of justifications for conflicts. New Directions for Child Development, 51, 71-86.

SMETANA, J. G. (1993). Conceptions of parental authority in divorced and married mothers and their adolescents. Journal of Research on Adolescence, 3(1), 19-39.

SMETANA, J. G. (1995). Parenting styles and conceptions of parental authority during adolescence. Child Development, 66, 299-316.

SMETANA, J. G.; y BERENT, R. (1993). Adolescents' and mothers' evaluations of justifications for disputes. Journal of Adolescent Research, 8(3), 252-273.

SMETANA, J. G.; y ASQUITH, P. (1994). Adolescents' and parents' conceptions of parental authority and personal autonomy. Child Development, 65, 1147-1162. 
SMETANA, J. G.; YAU, J.; y HANSON, S. (1991). Conflict resolution in families with adolescents. Journal of Research on Adolescence, 1(2), 189-206.

SMETANA, J. G.; YAU, J.; RESTREPO, A.; y BRAEGES, J. L. (1991). Adolescent-parent conflict in married and divorced families. Developmental Psychology, 27(6), 1000-1010.

SMETANA, J.; KILLEN, M.; y TURIEL, E. (1991). Children's reasoning about interpersonal and moral conflicts. Child Development, 62(3), 629-644.

SONG, M.; SMETANA, J.; y KIM, S. (1987). Korean children's conceptions of moral and conventional transgressions. Developmental Psychology, 23, 577-582.

TISAK, M. S.; y TURIEL, E. (1984). Children's conceptions of moral and prudential rules. Child Development, 55, 1030-1039.

TISAK, M.; y TURIEL, E. (1988). Variation in seriousness of transgressions and children's moral and conventional concepts. Developmental Psychology, 24(3), 352-357.

TURIEL, E. (1983). El conocimiento social. Moralidad y convención. Madrid: Debate, 1994.

TURIEL, E. (1989). Domain-specific social judgments and domain ambiguities. Merrill-Palmer Quarterly, 35, 89-114.

TURIEL, E., KILLEN, M.; y HELWIG, C. (1987). Morality: its structure, functions and vagaries. En J. Kagan; y S. Lamb (Eds.), The emergence of morality in young children (pp. 155-243). Chicago: University.

TURIEL, E.; HILDEBRANDT, C.; y WAINRYB, C. (1991). Judging social issues: difficulties, inconsistencies and consistencies. Monografs of the Society for Research in Child Development, 56(2), 1-103.

TURIEL, E.; NUCCI, L.; y SMETANA, J. (1988). A cross-cultural comparison about what? A critique of Nissan's (1987) study of morality and convention. Developmental Psychology, 24(1), 140-143.

TURIEL, E.; y DAVIDSON, Ph. L. (1986). Heterogeneity, inconsistency and asynchrony in the development of cognitive structures. En I. Levin (Ed.), Stage and structure. Norwood, N. J: Ablax.

WAINRYB, C. (1991). Understanding differences in moral judgments: the role of informational assumptions. Child Development, 62, 840-851.

WAINRYB, C. (1993). The application of moral judgments to other cultures: relativism and universality. Child Development, 64, 924-933.

WAINRYB, C.; y TURIEL, E. (1993). Conceptual and informational features in moral decision making. Educational Psychologist, 28(3), 205-218.

YAU, J.; y SMETANA, J. (1993). Chinese-american adolescent's reasoning about cultural conflicts. Journal of Adolescent Research, 8(4), 419-438. 